\title{
Reaction Physics and Mission Capabilities of the Magnetically Insulated Inertial Confinement Fusion Reactor
}

\author{
T. Kammash* and D. L. Galbraith $\dagger$ \\ University of Michigan, Ann Arbor, Michigan 48109
}

\begin{abstract}
The potential of the magnetically insulated inertial confinement fusion (MICF) reactor as a propulsion scheme is assessed using a quasi-one-dimensional, time-dependent set of equations to describe the plasma dynamics in the system. The target in this system consists of a spherical pellet whose inner wall is coated with deuterium-tritium fusion fuel, and the plasma is formed as a result of wall ablation by an incident laser beam that enters the pellet through a hole. In contrast to implosion-type inertial fusion, the plasma lifetime in MICF is much longer due to the fact that it is dictated by the shock speed in the metal shell that surrounds the fuel wall rather than by the sound speed in the plasma itself. By allowing the plasma at the end of the fusion burn to adiabatically expand into an expansion chamber then exhausting it through a magnetic nozzle, we determine the propulsion capabilities of such a system. For a reasonable pellet and chamber design we find that the propulsion capability of the system will allow a round trip to Mars to be made in a relatively short time.
\end{abstract}

\section{Introduction}

$\mathrm{N}$ a previous paper ${ }^{1}$ we introduced a fusion concept called the magnetically insulated inertial confinement fusion (MICF) reactor as a potentially attractive propulsion device for deep space missions and interplanetary travel. This system brings together the favorable aspects of both magnetic and inertial fusions in that physical containment of the burning plasma is provided by a metallic shell, and insulation of its thermal energy from material walls is provided by a strong, self-generated magnetic field as illustrated in Fig. 1. In constrast to conventional, implosion-type inertial confinement systems, MICF utilizes a plasma that is formed inside the target pellet as a result of ablation of the fuel-coated inner wall by a laser beam that enters the target through a hole. Since the confinement time in this system is dictated by the sound speed in the shell (as opposed to the sound speed in the plasma), a significant enhancement $\left(\sim 10^{2}\right)$ of the lifetime of plasma energy in MICF is achieved over implosion-type systems due in part to the reduction of the shell temperature arising from the thermal insulation provided by the magnetic field. Moreover, the coupling of laser energy to the plasma is more efficient in MICF since the energy is put directly into the plasma rather than in the imploding pusher as it is in implosion-type inertial fusion. The so-called Rayleigh-Taylor instability that has hampered conventional inertial fusion approaches does not arise in MICF due to the fact that in the magnetically insulated inertial fusion scheme, the light fluid (the plasma) is supported by the heavier fluid (the shell) against the gravitational force associated with the plasma acceleration, a situation known to be stable. Finally, a major benefit of this scheme lies in the fact that the expansion speed of the hot core is further reduced by the continuously ablative plasma from the solid fuel toward the central region. Since the ignition condition is dictated by the expansion speed of the hot core, it is evident that a significant reduction in this speed can allow for ignition at smaller input energies, perhaps on the order of few megajoules. Clearly, the most important figure of merit of any energy producing system is the energy multiplication or gain factor $Q$, which denotes the ratio of the energy produced by the fusion reactions to the energy put into the plasma to heat it to

Presented as Paper 88-2926 at the AIAA/ASME/SAE/ASEE 24th Joint Propulsion Conference, Boston, MA, July 11-13, 1988; received Nov. 3, 1988; revision received March 17, 1989. Copyright (C) 1988 by the American Institute of Aeronautics and Astronautics, Inc. All rights reserved.

*Professor, Department of Nuclear Engineering.

†Assistant Research Scientist, Department of Nuclear Engineering. thermonuclear temperatures. A $Q$ value of unity represents a break-even condition, and large $Q$ values are needed especially in systems where a meas? able amount of the power produced is required to operate the system itself. In the next section, we present a brief analysis of the dynamics of MICF and its performance as a propulsion device.

\section{Plasma Dynamics in MICF}

In order to fully assess MICF's propulsive capability, we utilize a quasi-one-dimensional, time-dependent set of particle and energy balance equations for the various species that constitute the plasma in this device. We use a deuterium-tritium (DT) fuel cycle and allow for two populations of the

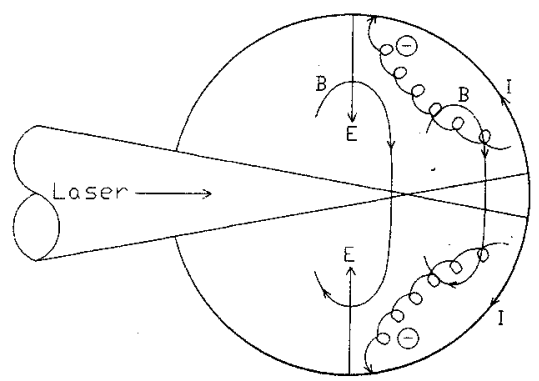

Fig. 1a MICF target pellet.

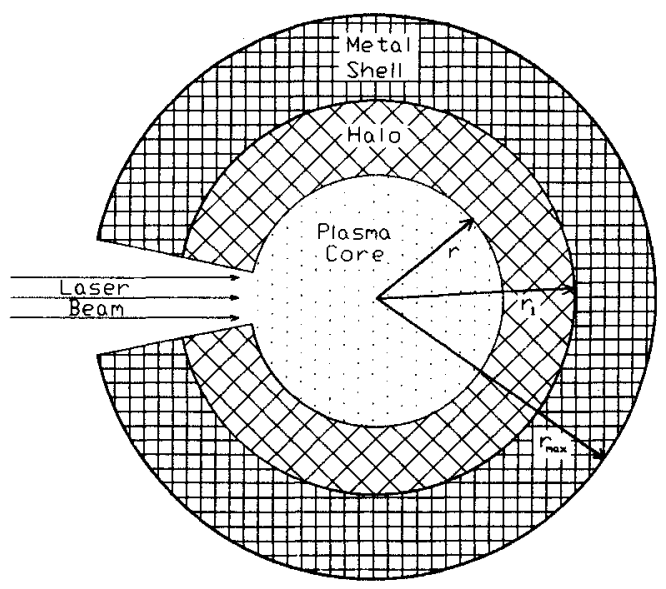

Fig. 1b Field generation in MICF. 
alpha particle generated by this fusion reaction, a fast alpha population born at $3.5-\mathrm{MeV}$ energy, and a thermal alpha population characterized by a Maxwellian distribution at an appropriate temperature. The details of the analysis can be found elsewhere, ${ }^{2,3}$ but for the purposes of this paper it is sufficient to note that the model we employ consists of a laser-ablative central region that is separated by a magnetic field from a "halo" region, which is adjacent to the solid fuel wall. A more realistic, but significantly more complex, model would consider the halo region to be surrounded by an ionized metal region, which itself is adjacent to a nonionized metal shell. We defer the adoption of such a model to a future calculation, but for the present, we choose the DT plasma to consist of three thermal species, namely the fuel ions, electrons, and thermal alphas in addition to an arbitrary number of fast alpha groups. We consider each species to be an ideal gas for which we draw upon thermodynamics to write the energy balance equation. The particle balance equation for the fuel ions (which we select to be one with a mass equal to the average masses of the deuteron and trition) is given by

$$
\frac{\mathrm{d}}{\mathrm{d} t}\left\{\frac{4}{3} \pi r^{3} n_{f}\right\}=-\frac{4}{3} \pi r^{3}\left\{\frac{1}{2} n_{f}^{2}<\sigma v>_{f}\right\}+4 \pi r^{2}\left\{\Gamma_{r}-\Gamma_{f}\right\}
$$

where $n_{f}$ denotes the ion density, $\langle\sigma v\rangle_{f}$ the velocity averaged fusion reaction cross section, which is temperature dependent; $\Gamma_{r}$ the particle flux for the refueling ions, which cross the magnetic field from the halo region to the core plasma; and $\Gamma_{f}$ represents the flux in the opposite direction. In Eq. (1), the first term on the right side reflects the loss of ions due to the fusion reaction, and the last term represents the source term arising from the net particle flux entering the core. The energy balance equations for the fuel ions can be expressed by

$$
\begin{aligned}
\frac{\mathrm{d}}{\mathrm{d} t} & \left\{\frac{(4 / 3) \pi r^{3}}{\gamma-1} n_{f} T_{f}\right\}+4 \pi r^{2} n_{f} T_{f} \frac{\mathrm{d} r}{\mathrm{~d} t}=\frac{4}{3} \pi r^{3} \\
& \left\{\frac{3}{2} \frac{n_{f} n_{e}}{(n \tau)_{e f}}\left(T_{e}-T\right)_{f}+\frac{3}{2} \frac{n_{f} n_{\alpha}}{(n r)_{\alpha f}}\left(T_{\alpha}-T_{f}\right)\right. \\
& \left.+n_{f} \sum_{k=1}^{k_{\max }} n_{k}\left(\frac{\mathrm{d} E_{k}}{\mathrm{~d} t}\right)\right\} \\
& -\frac{3}{4} n_{f}^{2}<\sigma v>_{f}+4 \pi r^{2}\left\{W_{r}-W_{f}\right\}
\end{aligned}
$$

The left side of Eq. (2) represents the change in the plasma thermal energy including the spherical expansion term. The first term on the right side denotes the energy exchange between the electrons and fuel ions and is characterized by the energy exchange constant $(n \tau)_{e f},{ }^{4}$ the second term represents the exchange with the thermal alpha particles, and the third term reflects the rate at which the $k$ th fast alpha group loses energy to the fuel ions. The fourth term denotes the energy removed from the ions due to their participation in the fusion reaction, and the last term represents the net energy flux between the refueling ions and ions escaping from the core. Similar equations are given for the other species in both regions but will not reproduced here in the interest of space.

As we noted earlier, the plasma in the core of MICF is kept away from the solid wall of the pellet by a strong magnetic field. Particle and energy transport take place in both directions across this field as we have observed in Eqs. (1) and (2). in the absence of turbulence, such diffusion takes place classically, i.e., as a result of Coulomb collisions between the species. We ignore this effect for the fast alpha particles since they tend to slow down more rapidly than they diffuse across the field. Moreover, we postulate for the purposes of this calculation that the bremsstrahlung emitted by the hot core electrons get absorbed primarily in the solid fuel wall, cause it to ablate, and contribute to the formation of the halo region. It is likely, however, that measurable photoionization can take place in the metal wall resulting in significant contribution to the back pressure exerted on the core plasma, but, once again, these details will be left to future studies. For the problem at hand, we contend that the radiation emitted by the hot electrons is the sole cause of the refueling mechanism and that for each photon absorbed, a single electron escapes from the wall without giving up an appreciable portion of its energy. Electrostatic effects then cause an equal number of positive ions to be emitted by the wall and to join the halo region. This fueling scheme may be viewed as somewhat optimistic since in a target with reasonably thin DT wall, a considerable fraction of the bremsstrahlung photons may indeed reach the metal shell and cause it to vaporize and ionize. But since, for the purposes of this paper, we are also ignoring other ablation mechanisms such as those due to ion heat wave, we proceed on the assumption that the above-described fueling mechanism is adequate. The number of fast alpha groups is chosen to be 10 , and the results were not significantly altered when a larger number of groups was utilized.

The stability of the plasma in the various regions of MICF plays an important role in the performance of the system as a reactor. The most likely instabilities are the drift modes associated with the density and temperature gradients in the system, ${ }^{5}$ but because the pressure is nearly constant across the transition region, ${ }^{6}$ the only instability of concern is the densitydriven electrostatic mode. The associated turbulence was incorporated in the analysis by adding a Bohm-like diffusion coefficient to represent the enhanced diffusion across the magnetic field in both directions. In many instances, this turbulence results in a more efficient fueling of the hot plasma which in turn manifests itself in a higher gain factor, $Q$.

As an illustration of MICF's performance as a reactor, we have chosen as a target a pellet with an outer radius of $0.75 \mathrm{~cm}$ and an inner metal shell radius of $0.375 \mathrm{~cm}$. The inner radius of the solid DT fuel is $0.25 \mathrm{~cm}$ and thus has a thickness of $0.125 \mathrm{~cm}$. For an incident laser energy of $3 \mathrm{MJ}$, a hot plasma core with a density of $2.5 \times 10^{21} \mathrm{~cm}^{-3}$ and a temperature of about $32 \mathrm{keV}$ is formed. Such a plasma was found to ignite resulting in a $Q$-value of 302 and was allowed at the end of the fusion burn to expand in a chamber of radius $10 \mathrm{~cm}$ with an exhaust hole of $2.50 \mathrm{~cm}$. The escape time for both the fuel ions and the metal ions is computed, and it is shown that a maximum firing rate of about 2700 can be accommodated. We denote the rep rate by " $\omega$ " and choose it to be 100 for the examples we present. It has been suggested ${ }^{7}$ that the mass of a laser driver with $2 \mathrm{MJ}$ of output energy is 262 metric tons (mT). By linear extrapolation, we suggest that the mass of a $3.0 \mathrm{MJ}$ laser system would be $393 \mathrm{mT}$ and for a $Q$ of 302 , the specific power of the system would be about $230 \mathrm{~W} / \mathrm{g}$-a very attractive value indeed.

\section{MICF as a Propulsion Device}

We proceed to examine the capabilities of MICF as a propulsion system. For that purpose we consider the case of the pellet design given in Table 1.

If such a pellet is housed in a combustion chamber with a radius of $10 \mathrm{~cm}$ and an opening of $2.50 \mathrm{~cm}$, which may be viewed as part of a magnetic nozzle with near-perfect efficiency, then by allowing the plasma (i.e., the charged particles) to adiabatically expand at the end of the fusion burn and then escape through the nozzle, we can compute, as noted earlier, the exhaust time, which in turn dictates the maximum repetition rate allowed. We have seen that $\omega=100$ is well within that limit. The propulsion capability of MICF can be estimated from the usual propulsion parameters that are determined from the standard rocket equations.. ${ }^{2}$ These parameters are the specific impulse, $I_{\mathrm{sp}}$; the thrust, $F$; and/or the jet power, which for the case at hand are summarized in Table 2.

It may be instructive to see how the parameters listed in Table 2 are obtained, particularly if we wish to examine those aspects of pellet design that would allow for a tradeoff between specific impulse and thrust. For the pellet configuration 
Table 1 Pellet design for propulsion

\begin{tabular}{ll}
\hline Inner radius of solid fuel, cm & 0.25 \\
Outer radius of solid fuel, cm & 0.375 \\
Outer radius of metal shell, cm & 0.750 \\
Hot plasma core density, cm -3 & $2.5 \times 10^{21}$ \\
Hot plasma core temperature, keV & 32 \\
Input energy, MJ & 3 \\
Gain factor & 302 \\
Total energy output, MJ & 906 \\
Charged particle energy, MJ & 181 \\
Total mass of pellet, g & 23.63 \\
\hline
\end{tabular}

Table 2 Propulsion parameters for an MICF system

\begin{tabular}{ll}
\hline Ion exhaust velocity, $\mathrm{km} / \mathrm{s}$ & 122 \\
Specific impulse, $I_{\mathrm{sp}}, \mathrm{s}$ & $1.25 \times 10^{4}$ \\
Repetition rate, $\omega, \mathrm{s}^{-1}$ & $\leq 2705$ \\
Thrust, $F, \mathrm{kN}$ & $0.34 \omega$ \\
Jet power, $P_{\text {jet }}, \mathrm{MW}$ & $2.64 \omega$ \\
\hline
\end{tabular}

Table 3 MICF rocket performance

\begin{tabular}{lccccc}
\hline \hline \multicolumn{5}{c}{$I_{\mathrm{sp}}=1.25 \times 10^{4} \mathrm{~s}, \dot{M}_{p}=2.363 \mathrm{~kg} / \mathrm{s}, M_{f}=869 \mathrm{mT}$} \\
Mission & $D_{\mathrm{AB}}(\mathrm{a} . \mathrm{u})$ & $R_{M}=1 / \alpha \beta$ & $M \mathrm{t}(\mathrm{m})$ & $M \mathrm{~T}(\mathrm{mT})$ & $\tau_{\mathrm{RT}}$ (days) \\
\hline Mars & 0.52 & 3.58 & 3,111 & 2,242 & 11 \\
Jupiter & 4.20 & 12.44 & 10,810 & 9,941 & 49 \\
\hline
\end{tabular}

described in Table 1, namely one where the solid fuel thickness is $0.125 \mathrm{~cm}$, and the metal shell thickness is $0.375 \mathrm{~cm}$, we take the fuel to be $50 \%-50 \%$ mixture of deuterium and tritium ions represented by an ion with an average mass of $2.5 \mathrm{amu}$. We take the metal shell to be made of tungsten whose density is $17.7 \mathrm{gram}-\mathrm{g} / \mathrm{cm}^{3}$ and whose atomic number is 74 . If we assume at the end of the fusion burn that the tungsten component of the pellet becomes vaporized, fully ionized, and in thermal equilibrium with the plasma, then we can readily calculated the number of particles and their average energy at that stage. The number of fuel ions will be $7.772 \times 10^{21}$, the number of tungsten ions will be $7.731 \times 10^{22}$, and the number of electrons will be $5.729 \times 10^{24}$. With an input energy of $3 \mathrm{MJ}$ and energy multiplication factor of 302 , the total energy produced, as displayed in Table 1 , is $906 \mathrm{MJ}$ or about $5.664 \times 10^{24}$ $\mathrm{keV}$. In the final state of the pellet-ignoring the electrons-

$$
\begin{aligned}
\text { Total mass } & =(2.5)\left(7.772 \times 10^{21}\right)+(183.86)\left(7.731 \times 10^{22}\right) \\
& =1.423 \times 10^{25} \mathrm{amu} \\
& =23.634 \mathrm{~g}
\end{aligned}
$$

If we further assume total mixing in that state, then the average energy per particle is obtained by dividing one-fifth the total energy by the total number of particles. Only onefifth of the energy appears in charged particles since fourfifths of the energy in a DT fusion reaction appears in the neutrons, which are assumed to instantly escape the chamber into the surroundings. In that case, the energy per charged particle becomes $1.948 \times 10^{-1} \mathrm{keV}$, and using the familiar expression for the kinetic energy, we find that the fuel ion exhaust velocity is $1.222 \times 10^{7} \mathrm{~cm} / \mathrm{s}$, and that of the tungsten ion is $1.425 \times 10^{6} \mathrm{~cm} / \mathrm{s}$. Clearly, the fuel ion exhaust velocity dictates the specific impulse generated by this propulsion device, and dividing this velocity by the Earth's gravitational acceleration, we obtain the specific impulse given in Table 2.

The thrust $F$ produced by MICF can be written as

$$
F=\left\{\sum_{j} m_{j} N_{j} v_{j}\right\} \omega
$$

where $m_{j}, N_{j}$, and $v_{j}$ are, respectively, the mass, number, and velocity of the $j$ th species, and $\omega$ is the rate at which the pellets are fired, i.e., the rep rate and the sum are over the species involved. Because of their small mass, the electron contribution is once again ignored whereas those of the fuel ions and tungsten ions are given, respectively, by $3.941 \times 10^{-3} \omega$ and $3.362 \times 10^{-1} \omega$. Once again, one of the species, namely the tungsten, is the primary contributor to the thrust, due to its larger mass, and the result is $F=0.34 \omega \mathrm{kN}$ as shown in Table 2. We conclude from this brief analysis that a pellet with a larger metallic component would be more desirable if the device is called upon to deliver large thrust whereas a pellet with a larger proportion of fusion fuel would be more appropriate when higher specific impulse in needed.

\section{Mars Missions}

As an interesting application to MICF as a propulsion device, we consider a round trip from Earth to Mars using the vehicle alluded to earlier. Without payload, the vehicle including the driver (lasers, radiators, optics, etc.), the thrust chamber, and the overhead, which includes the payload shield and other components, has a mass of $617 \mathrm{mT}^{7}{ }^{7}$ If we consider a piloted mission that includes a science payload and other relevant cargo of $252 \mathrm{mT}$, then the total vehicle mass would be $869 \mathrm{mT}$. Such a payload mission starting from low Earth orbit and using chemical propulsion and aerodynamic braking, and utilizing a split option trajectory profile ${ }^{8}$ will take about a year including a one month stay on that planet.

For our case, we will consider a round trip, continuous burn, acceleration/deceleration trajectory profile which assumes constant $I_{\mathrm{sp}}, F$, and $P_{\text {jet }}$ operation and take for the dry mass of the vehicle $869 \mathrm{mT}$ that includes a payload of $252 \mathrm{mT}$. The equations that describe the transit times for the outbound and return legs of the journey, $\tau_{\mathrm{RT}}$, from Earth (E) to Mars (M), along with the distances traveled $D_{\mathrm{EM}}$ and $D_{\mathrm{ME}}$ are given $\mathrm{by}^{9}$

$$
\begin{gathered}
\tau_{\mathrm{RT}}=\tau_{\mathrm{EM}}+\tau_{\mathrm{ME}}=\frac{I_{\mathrm{sp}}}{F / W_{f}}\left(\frac{1}{\alpha \beta}-1\right) \\
D_{\mathrm{EM}}=\frac{g I_{\mathrm{sp}}^{2}}{F / W_{f}}\left(\frac{1}{\beta}\right)\left(\frac{1}{\sqrt{\alpha}}-1\right)^{2} \\
D_{\mathrm{ME}}=\frac{g I_{\mathrm{sp}}^{2}}{F / W f}\left(\frac{1}{\sqrt{\beta}}-1\right)^{2} \\
\frac{1}{\alpha}=\frac{M_{i}}{M_{B}}, \frac{1}{\beta}=\frac{M_{B}}{M_{f}}, M_{B}=M_{f}+M_{p}^{B \rightarrow A}
\end{gathered}
$$

where $W_{f}=g M_{f}$ is the dry weight of the vehicle, $M_{i}$ the initial mass, and $M_{p}$ the propellant mass. Using the linear distance from Earth to Mars as 0.52 a.u. ( 1 a.u. $\left.\cong 1.5 \times 10^{11} \mathrm{~m}\right)$, we calculate $\alpha$ and $\beta$ from Eqs. (4) and (5), which upon substitution in Eq. (3) yields the round-trip time. Alternatively, the trip time can be computed from $M_{p} / \dot{M}_{p}$ where $\dot{M}_{p}$ represents the rate of flow of the propellant obtained by multiplying the pellet mass shown in Table 1 by the rep rate, $\omega$, which we take to be 100 . For purposes of comparison, we repeat the calculation for a round trip to Jupiter using the same vehicle and payloads. The results are shown in Table 3.

\section{Conclusions}

We have demonstrated in this study the unique capabilities of the MICF reactor as a propulsion system. A detailed investigation of the physics issues has revealed that fusion ignition can occur in MICF at moderate input energies, and very large gain factors can be obtained which translate into large exhaust velocities and very high specific impulses. In addition, large thrust and jet powers can be generated due to the presence of the metallic shell that surrounds the fusion fuel in the target pellets, and no serious confinement issues arise from such an arrangement. Although no effort was made to optimize the 
system, it is shown that with MICF propulsion, round-trip journeys to Mars and Jupiter can be made in 11 and 49 days, respectively, with substantial payloads on board. It must be noted, however, that these results were obtained on the assumption that the specific impulse is "generated solely by the fusion plasma. If the ions of the metal shell are included, the specific impulse will be degraded and the trip time will increase significantly.

\section{Acknowledgment}

This work was supported in part by the Department of Energy under Contract De-FG02-87 ER 13723. The authors are grateful to one of the reviewers for suggesting the present title of the paper and to $\mathrm{H}$. Buning for many useful discussions.

\section{References}

${ }^{1}$ Kammash, T., and Galbraith, D. L., "A Novel Fusion Scheme for Space Propulsion," AIAA Paper 87-2154, June 1987.

${ }^{2}$ Kammash, T., and Galbraith, D. L., "A Fusion Reactor for Space Applications," Fusion Technology, Vol. 12, No. 11, 1987.
${ }^{3}$ Kammash, T., and Galbraith, D. L., "Reactor Potential of the Magnetically Insulated Inertial Confinement Fusion System," Proceedings of the International Conference on Fusion Energy and Plasma Physics, World Scientific, NJ, 1987.

${ }^{4}$ Kammash, T., Fusion Reactor Physics, Principles and Technology, Ann Arbor Science Publishers, Ann Arbor, MI, 1975, Chap. 4.

${ }^{5}$ Aydemire, A. Y., Berk, H. L., Mirnov, V., Pogutse, O. P., and Rosenbluth, M. N., "Linear and Nonlinear Description of Drift Instabilities in a High Beta Plasma," Physics of Fluids, Vol. 30, 1987, p. 3038.

${ }^{6}$ Hasegawa, A., Nishihara, K., Daido, H., Fujita, M., Ishizaki, R., Miki, F., Mima, K., Murakami, M., Nakai, S., Terai, K., and Yamanaka, C., "Magnetically Insulated Inertial Fusion: A New Approach to Controlled Thermonuclear Fusion," Physical Review Letters, Vol. 56, 1986, p. 139.

7Hyde, R. A., "A Laser Fusion Rocket for Interplanetary Propulsion," Lawrence Livermore National Lab., Livermore, CA, Rept. UCRL-88856, 1983.

${ }^{8}$ Palaszewski, B., "Advanced Propulsion for Manned Lunar and Mars Missions," Jet Propulsion Lab., Pasadena, CA, Nov. 1987.

${ }^{9}$ Borowski, S. K., " A Comparison of Fusion/Anti-proton Propulsion Systems for Interplanetary Travel," AIAA Paper 87-1814, June 1987. 